Supporting Information

\title{
The Influence of the Strength of Drug-Polymer Interactions on the Dissolution of Amorphous Solid Dispersions
}

Kweku K Amponsah-Efah ${ }^{1}$, Pinal Mistry ${ }^{1,2}$, Reed Eisenhart ${ }^{3}$, Raj Suryanarayanan ${ }^{1 *}$

1. Department of Pharmaceutics, University of Minnesota, MN, USA

2. Current address: Pharmaceutical Sciences, AbbVie, Inc., Irvine, CA, USA

3. Department of Chemistry, University of Minnesota, MN, USA

*Correspondence:

Department of Pharmaceutics, College of Pharmacy, University of Minnesota, 308 Harvard Street SE, Minneapolis, MN 55455, USA.

E-mail: surya001@umn.edu 
(a)

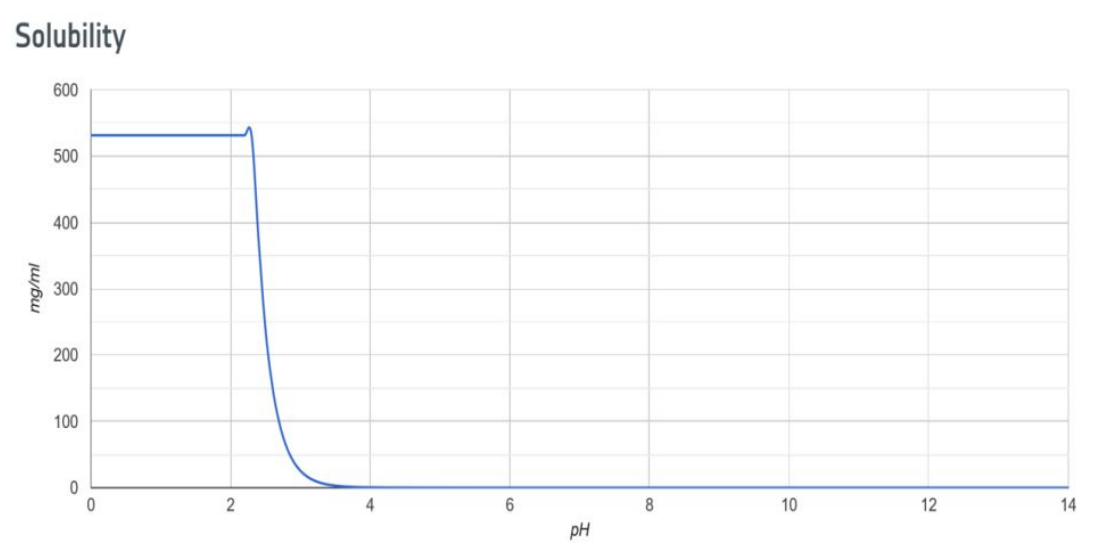

(b)

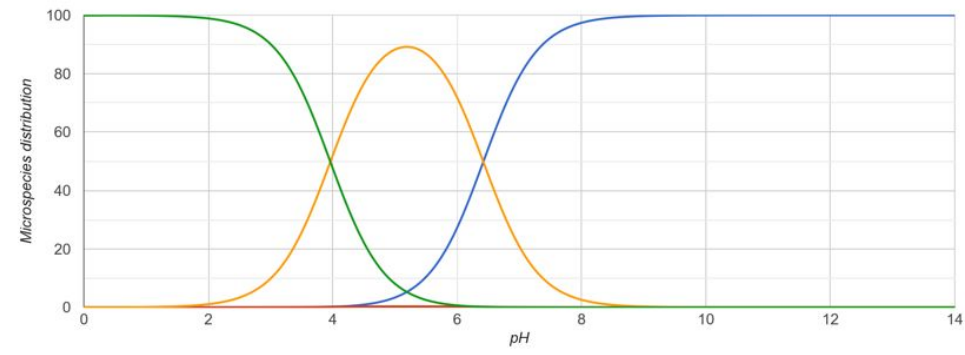

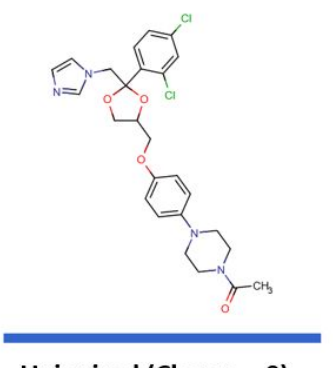

Unionized (Charge $=0)$

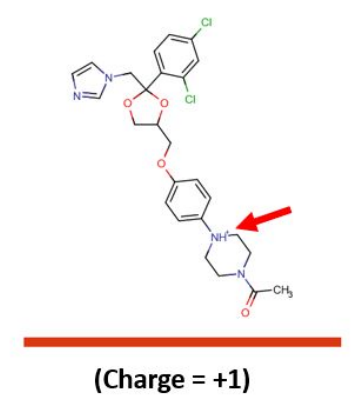

(Charge $=+1)$

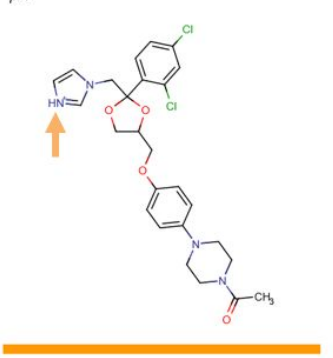

(Charge $=+1)$

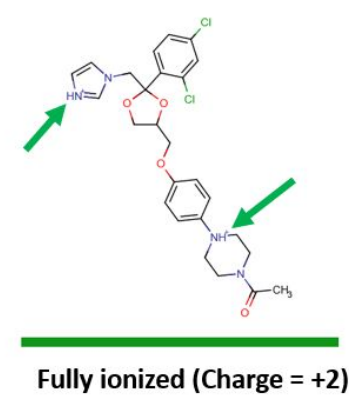

Figure S1. (a) Solubility of ketoconazole as a function of $p H$. (b) Microspecies distribution of ketoconazole at different aqueous solution $\mathrm{pH}$ values. Protonated regions are pointed out. Calculations (and output figures) were performed in Chem $3 D$ ultra ${ }^{\circledR}$. 


\section{Dissolution Experiments}

\section{(a) KTZ-PHEMA ASDs}

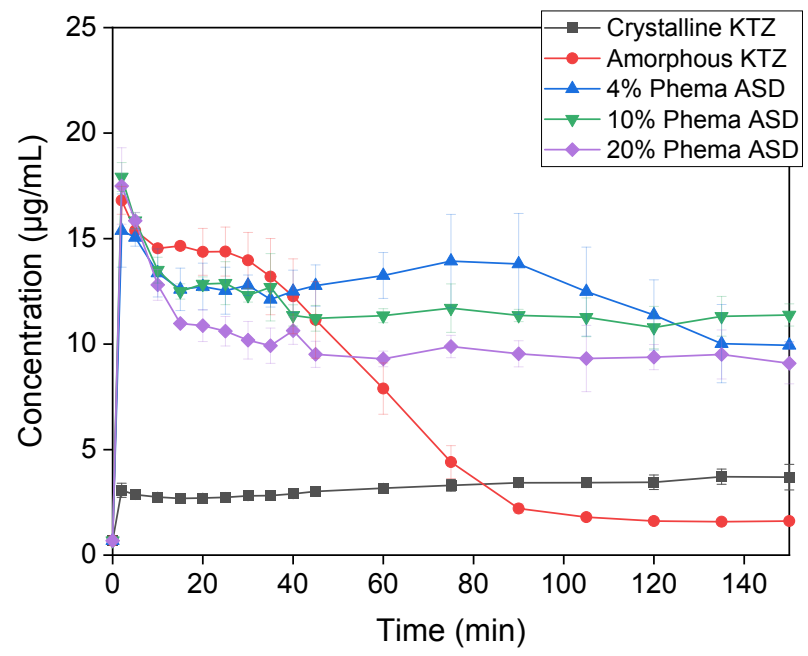

(b) KTZ-PHEMA Physical Mixtures

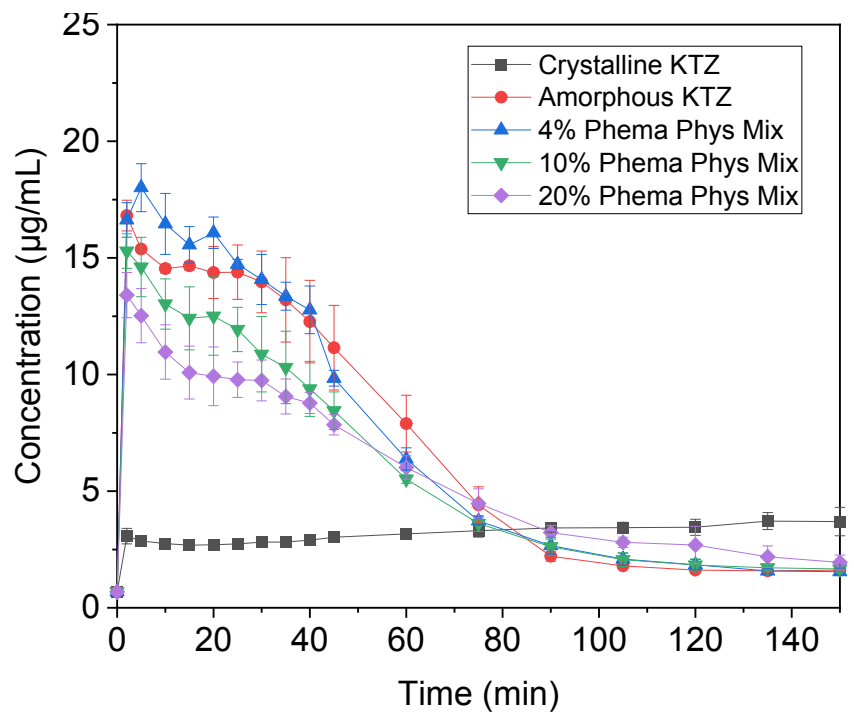

Figure S2. Dissolution profiles of crystalline KTZ, amorphous KTZ, and (a) ASDs or (b) Physical Mixtures, formulated with PHEMA, at polymer contents ranging between 4 and $40 \% \mathrm{w} / \mathrm{w}$ (mean \pm standard dev; $n=3$ ). Experiments were in performed in $\mathrm{pH} 7.4$ phosphate buffer at $37^{\circ} \mathrm{C}$. 


\section{Dissolution Experiments}

(a)

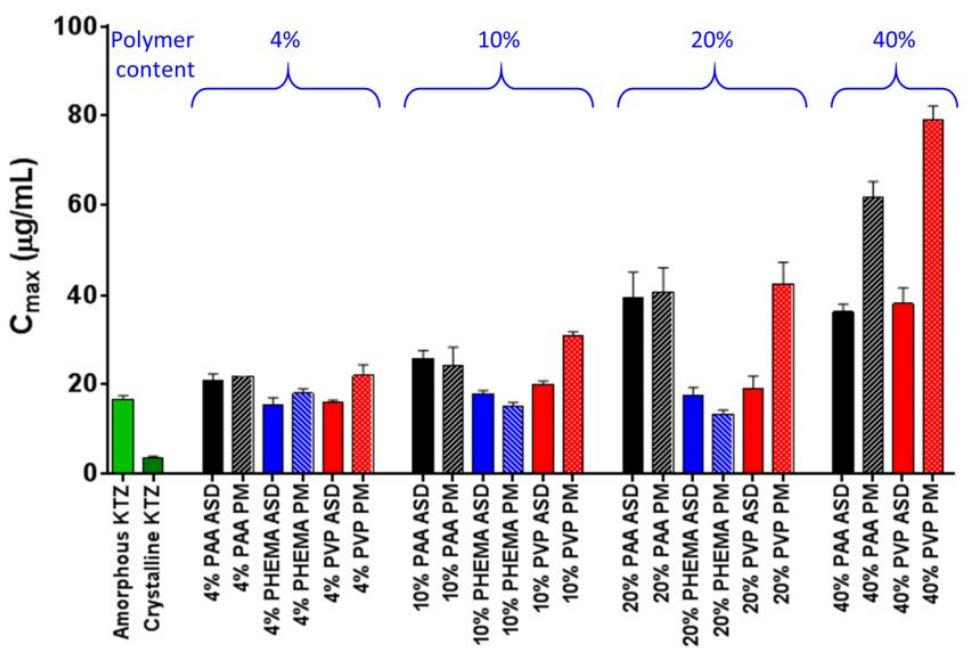

(b)

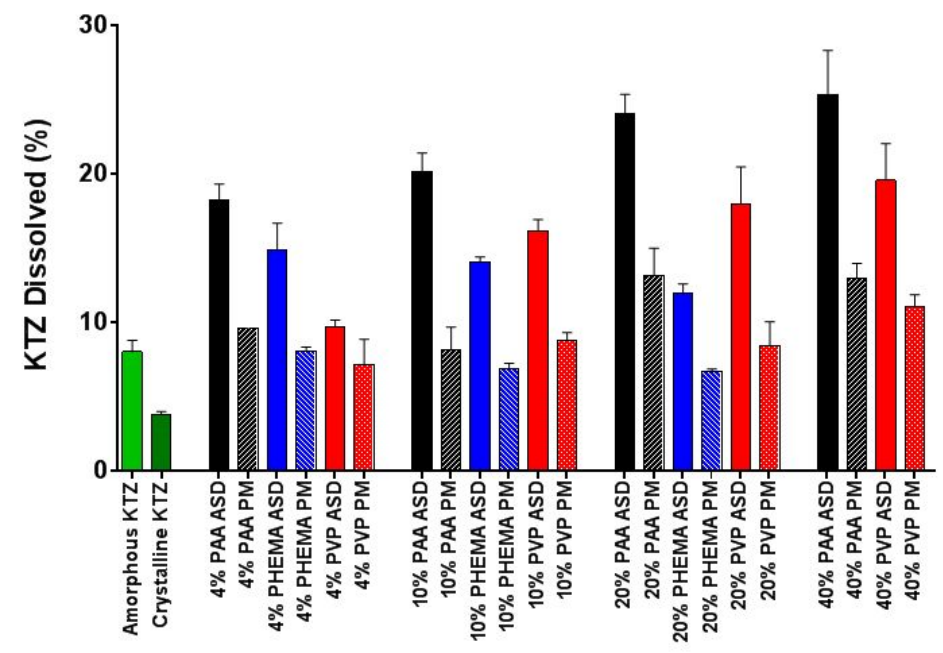

Figure S3. (a) Maximum dissolved drug concentration $\left(C_{\max }\right)$ values, (b) cumulative percent drug dissolved*, obtained from dissolution profiles of amorphous solid dispersions (ASDs) and physical mixtures (PMs), prepared with different polymers (PAA, PHEMA or $P V P)$ at polymer contents ranging between 4 and $40 \% \mathrm{w} / \mathrm{w}$. Values for crystalline and amorphous KTZ are included for comparison.

$* \mathrm{KTZ}$ Dissolved $\%=\left(\mathrm{AUC}_{0 \rightarrow 150 \mathrm{~min}} \times\right.$ Flow rate $) /$ Nominal drug weight $\times 100$ 


\section{D proton NMR}

Table S1. NMR peak assignments for $\mathrm{KTZ}$ in $\mathrm{D}_{2} \mathrm{O}$, acidified to $\mathrm{pH}$ 2.5. Based on structure numbering scheme of Figure 1.

\begin{tabular}{|l|l|l|}
\hline Assignment & Group & Chemical shift (ppm) \\
\hline H3 & Dichlorophenyl ring & doublet at 7.58 \\
\hline H5 & Dichlorophenyl ring & doublet of doublets at 7.38 \\
\hline H6 & Dichlorophenyl ring & doublet at 7.62 \\
\hline H8 & CH$_{2}$ link & AB quartet at 4.75, 4.85 \\
\hline H10 & Imidazole ring & single resonance at 8.68 \\
\hline H12 & Imidazole ring & triplet at 7.16 \\
\hline H13 & Imidazole ring & triplet at 7.38 \\
\hline H15 & Dioxolane & multiplet within 3.85 and 4.00 \\
\hline H16 & Dioxolane & doublet of doublets at 3.38 and 3.77 \\
\hline H18 & CH2 (methoxy) link & $\sim 4$ (doublet of doublets) \\
\hline H21, H25 & Para substituted phenyl ring & AA'BB' centered at 7.06 \\
\hline H22, H24 & Para substituted phenyl ring & AA'BB' centered at 6.85 \\
\hline H27, H31 & Piperazine ring & pair of triplets at 3.04 and 3.09 \\
\hline H28, H30 & Piperazine ring & Nevrlapping pair of doublets of doublets \\
\hline H33 & N-acetyl methyl 64 and 3.67 \\
\hline
\end{tabular}




\section{Nuclear Overhauser Effect Spectroscopy (NOESY)}

(a) Neat PAA

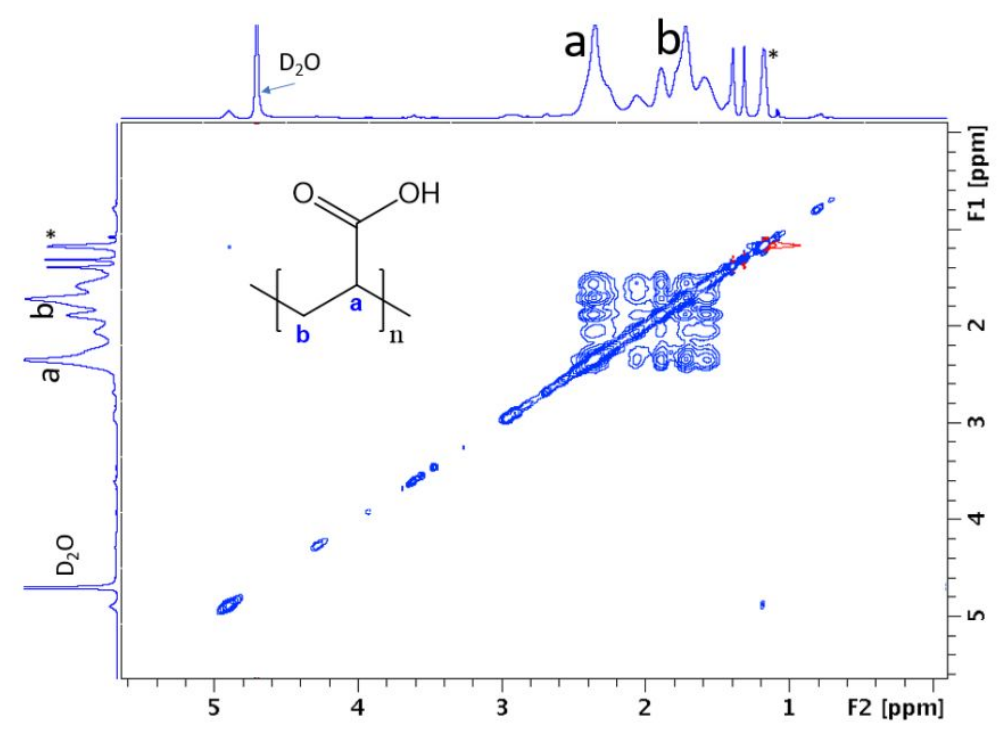

(b) Neat PVP

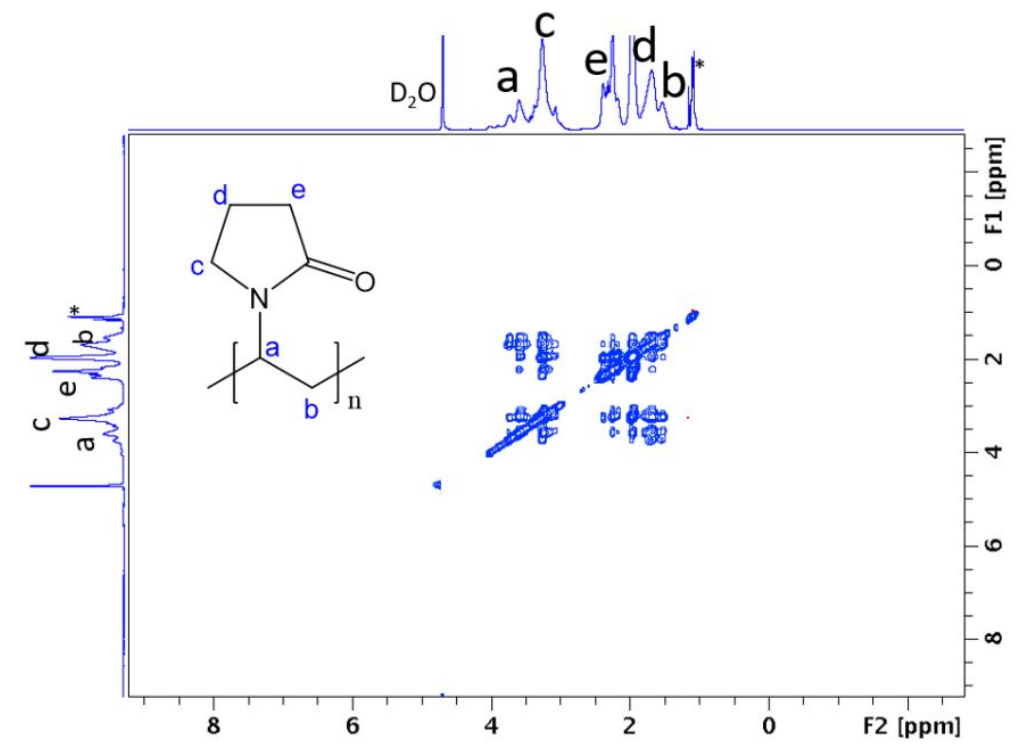

Figure S4. 2D ${ }^{1} H^{1} H$ NOESY spectra of (a) neat PAA and (b) neat PVP. Cross peaks are same-phased with the diagonal peaks (blue, negative sign). *Possibly from leached syringe material. 


\section{Diffusion Ordered Spectroscopy (DOSY)}

(a) KTZ alone

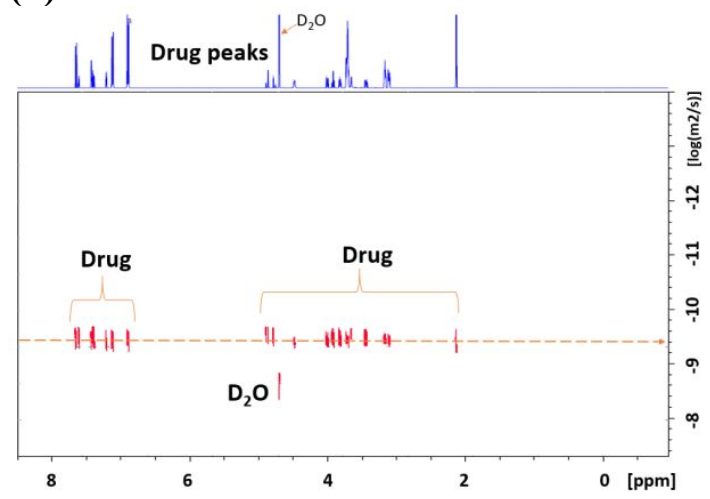

(c) PAA alone

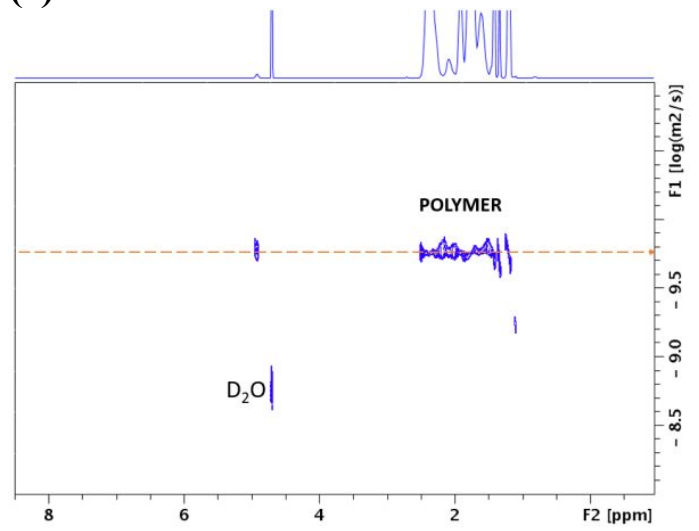

(e) $\mathrm{KTZ}+$ PAA

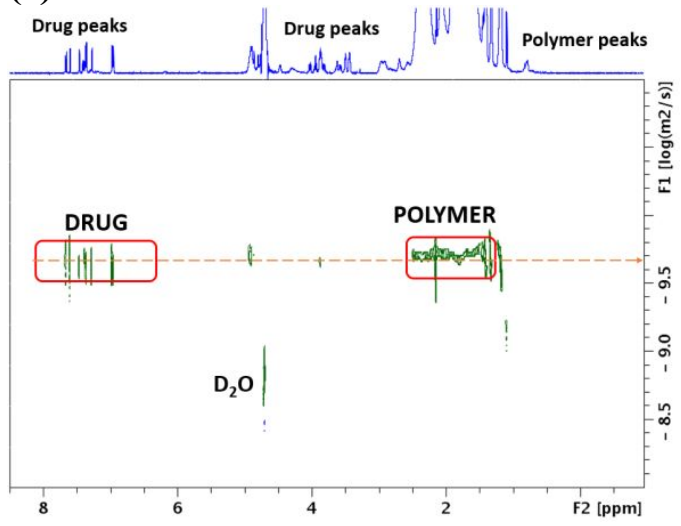

(b) Representative fit

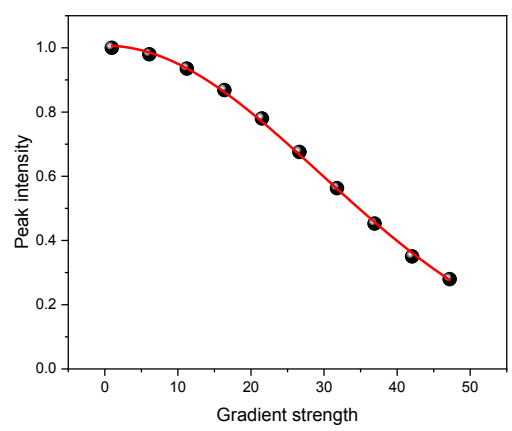

(d) PVP alone

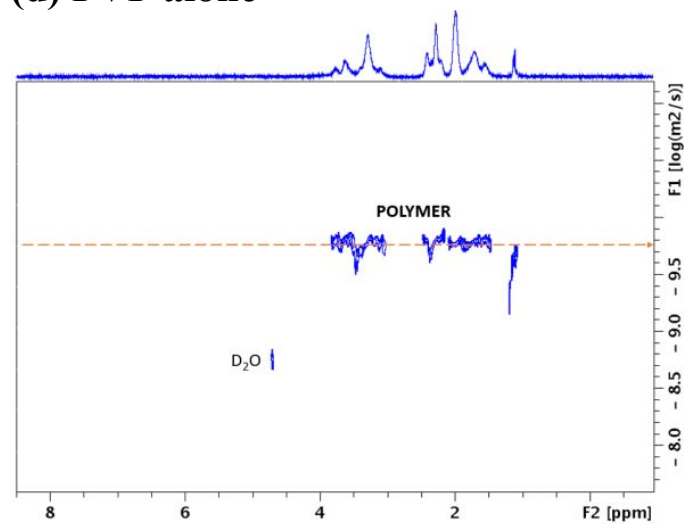

(f) KTZ + PVP

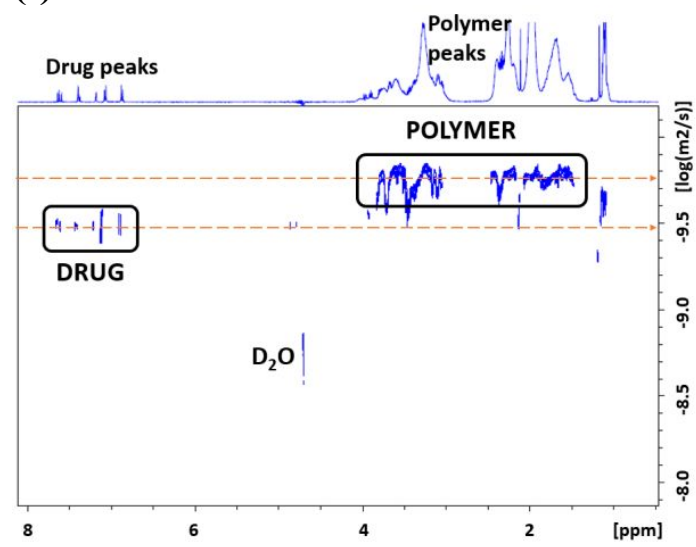

Figure S5 (a, c-f). Representative 2D DOSY plots of the neat drug (KTZ), the neat polymers (PAA or PVP), and the drug+ polymer mixtures. The solvent was $\mathrm{D}_{2} \mathrm{O}$ at pH 2.5. (b) Representative fit of equation (2) to the peak intensity vs gradient strength data. 
The DOSY results are displayed as a 2D matrix with the conventional $1 \mathrm{D}$ chemical shift spectrum plotted on the horizontal (top) axis, and the diffusion coefficients plotted along the perpendicular (right) axis. Diffusion peaks on each horizontal dashed line correlate with signals in the chemical shifts dimension, and are attributed to a specific molecular $\operatorname{species}^{53}$.

Table S2. Diffusion coefficients of neat KTZ, PAA and PVP (lines 1 to 3), and the drug or polymer in the drug+polymer mixtures (lines 4 to 7). Experiments were performed in $\mathrm{D}_{2} \mathrm{O}$ at $\mathrm{pH} 2.5$

\begin{tabular}{|r|l|r|r|}
\hline \multicolumn{1}{|c|}{$\boldsymbol{\#}$} & Sample & $\mathbf{D}\left(\mathbf{m}^{\mathbf{2}} \mathbf{s}^{-\mathbf{1}}\right) \times \mathbf{1 \mathbf { 0 } ^ { - 1 0 }} *$ & $\mathbf{D} / \mathbf{D}_{\mathbf{0}}$ \\
\hline 1 & KTZ alone & 3.548 & 1.00 \\
\hline 2 & PAA Alone & 1.778 & 0.50 \\
\hline 3 & PVP Alone & 1.769 & 0.50 \\
\hline 4 & KTZ in (KTZ + PAA) mixture & 2.042 & 0.58 \\
\hline 5 & PAA in (KTZ + PAA) mixture & 1.995 & 0.56 \\
\hline 6 & KTZ in (KTZ + PVP) mixture & 3.236 & 0.91 \\
\hline 7 & PVP in (KTZ + PVP) mixture & 1.715 & 0.48 \\
\hline 8 & D $_{2} \mathrm{O}$ & 17.780 & 5.01 \\
\hline
\end{tabular}

*Standard errors from fit of equation (2) to the intensity vs gradient strength data, on the order of $1 \times 10^{-12} \mathrm{~m}^{2} \mathrm{~s}^{-1}$ for all samples. 


\section{Isothermal Titration Calorimetry (ITC)}

$$
\begin{gathered}
{[\text { Drug }]+[\text { Polymer }] \stackrel{K_{a}}{\leftrightarrow}[\text { Drug } \cdot \text { Polymer }]} \\
\frac{d Q}{d[X]_{t}}=\Delta H^{o} V_{0}\left[\frac{1}{2}+\frac{1-X_{R}-r}{2 \sqrt{\left(1+X_{R}+r\right) 2-4 X_{R}}}\right]+Q_{d i l}
\end{gathered}
$$

Equation (S1) assumes as simple 1:1 interaction between the drug and polymer, with an association constant $K_{a}$. In equation (S2), $Q$ is the heat recorded, $[X]_{t}$ is the molar concentration of injectant at time $\mathrm{t}, \Delta H^{\circ}$ is the molar enthalpy change, $V_{0}$ is the initial volume of the sample cell, $X_{R}$ is the mole ratio of drug to polymer in the sample cell, and $Q_{d i l}$ is the molar enthalpy of dilution, an adjustable parameter included to account for the dilution of drug in the solvent. The term $r$ is defined as:

$$
\frac{1}{r}=n K_{a}[M]_{t}
$$

where $n$ is the stoichiometric ratio and $[M]_{t}$ is the molar concentration of polymer at time t. The Gibbs free energy change $(\Delta G)$, enthalpy change $(\Delta H)$ and entropy change $(\Delta S)$, can be calculated from the fundamental thermodynamic relation:

$$
\Delta G=-R T \ln K_{a}=\Delta H-T \Delta S
$$

where $R$ is the universal gas constant, and $T$ is temperature.

In the standard one-sites model, $n$, the stoichiometry number, represents the average number of drug binding sites per mole of macromolecule in the solution, with the 
assumption that all sites are identical and independent. In standard ITC fitting, the stoichiometry number can be obtained from the fit. However, for isotherms with weak affinity, (commonly termed low " $c$ " data), the stoichiometry number can be fixed at 1 , to reduce the number of variables, and increase the confidence in the fitted enthalpy change parameter. A global fit of the individual isotherms obtained before concatenation (see methods section for additional details) was performed, and the thermodynamic signature obtained was consistent with the values from the linear fit. 